Received: 22.01 .2019

Revised: 27.09.2019

Accepted: 25.10 .2019

DOI: $10.17804 / 2410-9908.2019 .5 .073-080$

\title{
ANALYSIS OF TWO CREEP RUPTURE MODELS
}

\author{
V. V. Nazarov \\ Institute of Mechanics, Lomonosov Moscow State University \\ 1 Michurinskiy Ave., Moscow, 119192, Russian Federation \\ iD https://orcid.org/0000-0002-9234-3646; $ه$ inmec130@mail.ru \\ Corresponding author. E-mail: inmec130@mail.ru \\ Address for correspondence: 1 Michurinskiy Ave., Moscow, 119192, Russian Federation
}

\begin{abstract}
Various invariants of the stress tensor (maximum normal stress, Mises equivalent stress, doubled maximum tangential stress) are considered, as well as their linear combinations with one material parameter, when approximating the experimental creep rupture data obtained under a complex stress state. The error of the total discrepancy between the experimental data and the approximating values is always less for linear combinations with the material parameter than for the basic invariants of the stress tensor. This determines the predominant practical use of these linear combinations with the parameter. In this paper, we consider two models for describing the creep-rupture process under a complex stress state. One is a linear combination of the Mises equivalent stress and the maximum normal stress. The other is a linear combination of the doubled maximum tangential stress and the maximum normal stress. The effect of each of the two maximum stresses on the rupture time is established from the analysis of the results of the statistical processing of experimental data obtained under tension and torsion of tubular specimens.
\end{abstract}

Keywords: creep rupture, rupture time, complex stress state, stress tensor invariant.

\section{References}

1. Lokoshchenko A.M., Nazarov V.V. Kinetic approach of investigation of creep-rupture for metals under biaxial tension. Aviatsionno-Kosmicheskaya Tekhnika i Tekhnologiya, 2005, no. 10 (26), pp. 73-79. (In Russian).

2. Lokoshchenko A.M. and Nazarov V.V. Choice of Long-Term Strength Criteria for Metals in Combined Stress State. Aerospace Engineering and Technology, 2004, no. 7 (15), pp. 124-128. (In Russian).

3. Lokoshchenko A.M. Estimation of Equivalent Stresses in the Analysis of Long-Term Strength of Metals under Combined Stress State. Mechanics of Solids, 2010, vol. 45, no. 4, pp. 633-647. DOI: $10.3103 / \mathrm{S} 0025654410040126$.

4. Lebedev A.A. The theory of equivalent stresses as a problem of mechanics of materials. Strength of Materials, 1996, vol. 28, no. 2, pp. 94-108. DOI: 10.1007/BF02215833.

5. Dyson B.F., Mclean D. Creep of Nimonic 80A in torsion and tension. Metal Science, 1977, vol. 11 , iss. 2, pp. 37-45. DOI: 10.1179/msc.1977.11.2.37.

6. Cane B.J. Creep damage accumulation and fracture under multiaxial stresses. In: Proc. 5th Int. Conf. Fract., Cannes, 1981, vol. 3, pp. 1285-93.

7. Nazarov V.V. Determination of creep properties under tension and torsion of copper tubular specimens. Inorganic Materials, 2014, vol. 50, no. 15, pp. 1514-1515. DOI: 10.1134/S0020168514150138.

8. Nazarov V.V. Description of Steady Creep under Tension and Torsion of Tubular Samples. Zavodskaya Laboratoriya. Diagnostika Materialov, 2015, vol. 81, no. 7, pp. 60-61. (In Russian). 
Подана в журнал: 22.01.2019

УДК 620.172.2:620.162.3:539.376

DOI: $10.17804 / 2410-9908.2019 .5 .073-080$

\title{
АНАЛИЗ ДВУХ МОДЕЛЕЙ ДЛИТЕЛЬНОЙ ПРОЧНОСТИ
}

\author{
В. В. Назаров
}

\author{
Научно-исследовательский институт механики \\ Федерального государственного бюджетного образовательного учреждения высшего образования \\ «Московский государственный университет им. М.В. Ломоносова» \\ Мичуринский проспект, 1, Москва, 119192, Российская Федерация \\ https://orcid.org/0000-0002-9234-3646; ه inmec130@mail.ru \\ Ответственный автор. Электронная почта: inmec130@ mail.ru \\ Адрес для переписки: Мичуринский проспект, 1, Москва, 119192, Российская Федерация
}

При аппроксимации опытных данных длительной прочности, полученных в условиях сложного напряженного состояния, рассматривают различные инварианты тензора напряжений (максимальное нормальное напряжение, интенсивность напряжений удвоенное максимальное касательное напряжение), а также их линейные комбинации с одним материальным параметром. Погрешность суммарного расхождения экспериментальных данных и аппроксимирующих значений всегда меньше для линейных комбинаций с материальным параметром, чем для базовых инвариантов тензора напряжений, что предопределяет преимущественное использование на практике этих линейных комбинаций с материальным параметром. В работе рассмотрены две модели описания процесса длительной прочности при сложном напряженном состоянии: первая (1) - линейная комбинация интенсивности напряжений и максимального нормального напряжения, вторая (2) - линейная комбинация удвоенного максимального напряжения сдвига и максимального нормального напряжения. Из анализа результатов статистической обработки экспериментальных данных, полученных при растяжении и кручении трубчатых образцов, установлено влияние каждого из двух максимальных напряжений на время разрыва, а также преимущество использования одной из двух этих моделей.

Ключевые слова: длительная прочность, время в момент разрушения, сложное напряженное состояние, инвариант тензора напряжений.

\section{1. Введение}

Для описания процесса длительной прочности при сложном напряженном состоянии используют два подхода [1, 2]. Кинетический подход [1] описывает процесс длительной прочности в условиях воздействия растягивающих главных напряжений. Главные напряжения, которые равны нулю или меньше нуля, не принимают участие в процессе накопления повреждений и не учитываются при описании процесса длительной прочности. Для главных напряжений $\sigma_{1}>\sigma_{2}>\sigma_{3}>0$ абсолютная величина вектора поврежденности $\omega(t)=\sqrt{\omega_{1}^{2}(t)+\omega_{2}^{2}(t)+\omega_{3}^{2}(t)}$, при этом в начальный момент времени $\left.\omega\right|_{t=0}=0$ и в момент времени разрушения $\left.\omega\right|_{t=t_{\text {rupt }}}=1$, где $t$ - время процесса ползучести. Скорости накопления компонент вектора повреждений пропорциональны соответствующим главным напряжениям: $d \omega_{1} / d t \sim \sigma_{1} ; d \omega_{2} / d t \sim \sigma_{2} ; d \omega_{3} / d t \sim \sigma_{3}$. При двухосном растяжении главные напряжения $\sigma_{1}>\sigma_{2}>0, \sigma_{3}=0$ и абсолютная величина вектора накопления повреждений 
принимает значение $\omega(t)=\sqrt{\omega_{1}^{2}(t)+\omega_{2}^{2}(t)}$. Тем не менее кинетический подход [1] бесполезен для описания процесса длительной прочности при растяжении и кручении трубчатых образцов, при котором главные напряжения $\sigma_{1}>0, \sigma_{2}=0, \sigma_{3}<0$. Между тем, критериальный подход [2,3] описывает процесс длительной прочности при таком сложном напряженном состоянии $\left(\sigma_{1}>0, \sigma_{2}=0, \sigma_{3}<0\right)$. Суть подхода $[2,3]$ заключается в том, что для аппроксимации времени разрушения используются различные инварианты тензора напряжений: максимальное нормальное напряжение $\sigma_{\max }$, интенсивность напряжений $\sigma_{\text {mises }}$, удвоенное максимальное касательное напряжение $2 \tau_{\max }$, а также их линейные комбинации с параметром. Все эти инварианты тензора напряжений при одноосном растяжении оказываются равными максимальному нормальному напряжению, что означает $\sigma_{\max }=\sigma_{\operatorname{mises}}=2 \tau_{\max }$. При сложнонапряженном состоянии инварианты тензора напряжений принимают различные значения, при этом линейные комбинации инвариантов тензора напряжений с параметром позволяют лучше описать процесс длительной прочности, чем базовые инварианты тензора напряжений $[2,3]$.

\section{2. Две рассматриваемые модели}

Из анализа [2, 3] погрешностей суммарного расхождения опытных данных, полученных в условиях совместного растяжения и кручения трубчатых образцов относительно аппроксимирующих значений, установлено, что из числа рассмотренных инвариантов тензора напряжений минимальная погрешность соответствует линейной комбинации $\sigma_{\text {inv }}^{1}[4]$

$$
\sigma_{\text {inv }}^{1}=\left[1-\beta_{1}\right] \sigma_{\text {mises }}+\beta_{1} \sigma_{\max }, \quad 0 \leq \beta_{1} \leq 1,
$$

где $\beta_{1}$ - параметр, учитывающий влияние интенсивности напряжений и максимального нормального напряжения на время в момент разрушения. В силу того, что интенсивность напряжений - только математическая характеристика тензора напряжений, а максимальное напряжение сдвига - реальная физическая величина, то взамен интенсивности напряжений аналогично (1) рассмотрим удвоенное максимальное напряжение сдвига

$$
\sigma_{\text {inv }}^{2}=\left[1-\beta_{2}\right]\left[2 \tau_{\max }\right]+\beta_{2} \sigma_{\max }, \quad 0 \leq \beta_{2} \leq 1,
$$

где $\beta_{2}$ - параметр, учитывающий влияние удвоенного максимального напряжения сдвига и максимального нормального напряжения на время в момент разрушения. Влияние инвариантов тензора напряжений на время в момент разрушения определяется значениями параметров $\beta_{1}$ и $\beta_{2}$ (табл. 1).

Таблица 1 - влияние различных инвариантов тензора напряжений на время в момент разрушения

\begin{tabular}{|c|c|c|c|}
\hline $\begin{array}{c}\text { Параметры } \\
\text { напряжений }\end{array}$ & $\sigma_{\max }$ & $2 \tau_{\max }$ & $\sigma_{\operatorname{mises}}$ \\
\hline $0 \leq \beta_{1}<0,5$ & Слабое влияние & - & Сильное влияние \\
\hline $0,5<\beta_{1} \leq 1$ & Сильное влияние & - & Слабое влияние \\
\hline $0 \leq \beta_{2}<0,5$ & Слабое влияние & Сильное влияние & - \\
\hline $0,5<\beta_{2} \leq 1$ & Сильное влияние & Слабое влияние & - \\
\hline
\end{tabular}




\section{3. Экспериментальные данные и методика статистической обработки}

Определим значения параметров $\beta_{1}$ и $\beta_{2}$ вместе с погрешностями $\Delta_{1}$ и $\Delta_{2}$ для выражений (1) и (2). Из анализа погрешностей можно определить преимущество одной из двух линейных комбинаций (1) и (2). С этой целью проведем статистическую обработку экспериментальных данных (табл. 2), полученных при растяжении и кручении трубчатых образцов. Между временем разрыва $t_{\text {rupt }}^{\text {approx }}$ и инвариантом $\sigma_{\text {inv }}$ тензора напряжений примем степенную связь с двумя материальными параметрами:

$$
\frac{t_{\text {rupt }}^{\text {approx }}}{t_{\text {dim }}}=B\left[\frac{\sigma_{\text {inv }}}{\sigma_{\text {dim }}}\right]^{-m}, \quad B>0, \quad m>0,
$$

где $\sigma_{\text {inv }}-\sigma_{\text {inv }}^{1}$ или $\sigma_{\text {inv }}^{2} ; t_{\text {dim }}=1$ ч и $\sigma_{\text {dim }}=1 \mathrm{MПа} \mathrm{-} \mathrm{произвольные} \mathrm{размерные} \mathrm{величины,} B$; $m$ - материальные параметры.

Таблица 2 - Экспериментальные данные для статистической обработки

\begin{tabular}{|c|c|c|c|c|c|}
\hline \multirow[b]{2}{*}{ Автор } & \multirow{2}{*}{$\begin{array}{c}\text { Металлический } \\
\text { материал }\end{array}$} & \multirow{2}{*}{$\begin{array}{c}\text { Высокая } \\
\text { температура, } \\
t,{ }^{\circ} \mathrm{C}\end{array}$} & \multicolumn{3}{|c|}{$\begin{array}{c}\text { Количество опытов для различных типов } \\
\text { стационарного нагружения }\end{array}$} \\
\hline & & & $\begin{array}{c}\text { Чистое } \\
\text { растяжение }\end{array}$ & $\begin{array}{l}\text { Чистое } \\
\text { кручение }\end{array}$ & $\begin{array}{l}\text { Совместное } \\
\text { растяжение } \\
\text { и кручение }\end{array}$ \\
\hline Dyson [5] & $\begin{array}{c}\text { Никелевый } \\
\text { сплав } \\
\text { Nimonic 80a }\end{array}$ & 750 & 8 & 9 & - \\
\hline Cane [6] & $\begin{array}{c}\text { Сталь } \\
2,25 \mathrm{Cr}-1 \mathrm{Mo}\end{array}$ & 565 & 6 & 4 & - \\
\hline Назаров [7] & $\begin{array}{c}\text { Отожженная } \\
\text { медь }\end{array}$ & 264 & 2 & - & 3 \\
\hline
\end{tabular}

Базовые инвариантные напряжения:

$$
\sigma_{\max }=\sigma_{1}, \quad \sigma_{\text {mises }}=\sqrt{\sigma_{1}^{2}-\sigma_{1} \sigma_{3}+\sigma_{3}^{2}}, \quad \tau_{\max }=\frac{\sigma_{1}-\sigma_{3}}{2} .
$$

Главные напряжения [8] при растяжении и кручении трубчатых образцов

$$
\sigma_{1}=\frac{\sigma}{2}+\sqrt{\left[\frac{\sigma}{2}\right]^{2}+\tau^{2}}, \quad \sigma_{2}=0, \quad \sigma_{3}=\frac{\sigma}{2}-\sqrt{\left[\frac{\sigma}{2}\right]^{2}+\tau^{2}},
$$

где $\sigma$ - нормальное напряжение; $\tau$ - касательное напряжение. Неизвестные параметры аппроксимации процесса длительной прочности определяются минимальной погрешностью, равной сумме расстояний экспериментальных точек относительно отрезка аппроксимирующей прямой в логарифмических осях

$$
\Delta=\min \left(\sum_{1}^{N}\left|\lg \frac{t_{\mathrm{rupt}}^{\text {approx }}\left(\sigma_{\mathrm{inv}}\right)}{\mathrm{t}_{\mathrm{rupt}}^{\mathrm{approx}}\left(\sigma_{\mathrm{inv}}\right)}\right|\right)
$$


где $N$ - количество опытных точек; $t_{\text {rupt }}^{\text {exper }}-$ время разрушения из эксперимента. Вычисления проводились методом Generalized Reduced Gradient (Microsoft Excel), при этом погрешности $\Delta_{1}$ и $\Delta_{2}$ использовались в качестве целевых условий минимального суммарного расхождения между экспериментальными данными и аппроксимирующими значениями.

\section{4. Анализ результатов}

Из анализа вычисленных параметров (табл. 3-4) следует, что для одной и той же серии испытаний они незначительно отличаются друг от друга. Разные значения параметра $\beta_{2}$ указывают на различное влияние максимальных напряжений на время в момент разрушения.

Таблица 3 - вычисленные параметры линейной комбинации $\sigma_{\text {inv }}^{1}$

\begin{tabular}{|c|c|c|c|c|}
\hline $\begin{array}{c}\text { Металлический } \\
\text { материал }\end{array}$ & $\begin{array}{c}\text { Высокая } \\
\text { температура } \\
t,{ }^{\circ} \mathrm{C}\end{array}$ & $\lg B_{1}$ & $m_{1}$ & $\beta_{1}$ \\
\hline $\begin{array}{c}\text { Никелевый } \\
\text { сплав } \\
\text { Nimonic 80a }\end{array}$ & 750 & 13,844 & 4,9 & 0,5 \\
\hline $\begin{array}{c}\text { Сталь } \\
\text { 2,25Сr-1Мо }\end{array}$ & 565 & 11,262 & 3,8 & 0,7 \\
\hline $\begin{array}{c}\text { Отожженная } \\
\text { медь }\end{array}$ & 264 & 7,712 & 3,0 & 0,0 \\
\hline
\end{tabular}

Таблица 4 - Вычисленные параметры линейной комбинации $\sigma_{\text {inv }}^{2}$

\begin{tabular}{|c|c|c|c|c|}
\hline $\begin{array}{c}\text { Металлический } \\
\text { материал }\end{array}$ & $\begin{array}{c}\text { Высокая } \\
\text { температура, } \\
t,{ }^{\circ} \mathrm{C}\end{array}$ & $\lg B_{2}$ & $m_{2}$ & $\beta_{2}$ \\
\hline $\begin{array}{c}\text { Никелевый } \\
\text { сплав } \\
\text { Nimonic 80a }\end{array}$ & 750 & 13,505 & 4,7 & 0,6 \\
\hline $\begin{array}{c}\text { Сталь } \\
\text { 2,25Сr-1Мо }\end{array}$ & 565 & 11,179 & 3,8 & 0,8 \\
\hline $\begin{array}{c}\text { Отожженная } \\
\text { медь }\end{array}$ & 264 & 7,812 & 3,0 & 0,0 \\
\hline
\end{tabular}

Этот эффект, скорее всего, не зависит от вклада нормального $\sigma$ и касательного $\tau$ напряжений в максимальное нормальное напряжение $\sigma_{\max }$ и удвоенное максимальное касательное напряжение $2 \tau_{\max }$. Это объясняется тем, что равенство $2 \tau_{\max }=\sigma_{\max }$ достигается либо при чистом растяжении $(\tau=0)$, либо при чистом кручении $(\sigma=0)$ трубчатого образца $[5,6]$. Неравенство $2 \tau_{\max }>\sigma_{\max }$ выполняется при совместном растяжении $(\sigma>0)$ и кручении $(\tau>0)$ трубчатого образца [7]. Неравенство $2 \tau_{\max } \geq \sigma_{\max }$ (это означает $\beta_{2}<0,5$ ) выполняется при любом сложном напряженном состоянии, при этом наблюдается противоречие $\beta_{2}>0,5$ для сплава Nimonic 80a и стали 2,25Cr-1Мо (табл. 4). Из анализа значений $\beta_{2}$ следует, что в экспериментах $[5,6]$ максимальное нормальное напряжение $\sigma_{\max }$ оказывает более существенное влияние на время разрушения, чем удвоенное максимальное касательное напряжение $2 \tau_{\max }$. Добавление 
параметра ( $\beta_{1}$ или $\left.\beta_{2}\right)$ в инвариант тензора напряжений, за исключением [7], приводит к уменьшению погрешности (табл. 5). Экспериментальные точки располагаются вдоль отрезка аппроксимирующей линии (рис. 1 и 2). Таким образом, модели 1 и 2 одинаково хорошо описывают процесс длительной прочности при сложном напряженном состоянии.

Таблица 5 - Погрешности суммарных расхождений опытных данных относительно аппроксимирующих значений для инвариантов тензора

напряжений $\sigma_{\max }, \sigma_{\text {mises }}, 2 \tau_{\max }, \sigma_{\text {inv }}^{1}, \sigma_{\text {inv }}^{2}$

\begin{tabular}{|c|c|c|c|c|c|c|}
\hline $\begin{array}{c}\text { Metallic } \\
\text { material }\end{array}$ & $\begin{array}{c}\text { High } \\
\text { temperature } \\
t,{ }^{\circ} \mathrm{C}\end{array}$ & $\Delta_{\sigma_{\max }}$ & $\Delta_{\sigma_{\text {mises }}}$ & $\Delta_{2 \tau_{\max }}$ & $\Delta_{1}$ & $\Delta_{2}$ \\
\hline Nimonic 80a & 750 & 4,3 & 4,3 & 6,6 & 1,6 & 1,7 \\
\hline $2,25 \mathrm{Cr}-1 \mathrm{Mo}$ & 565 & 1,6 & 2,5 & 3,2 & 0,7 & 0,7 \\
\hline Copper & 264 & 0,6 & 0,5 & 0,3 & 0,5 & 0,3 \\
\hline
\end{tabular}
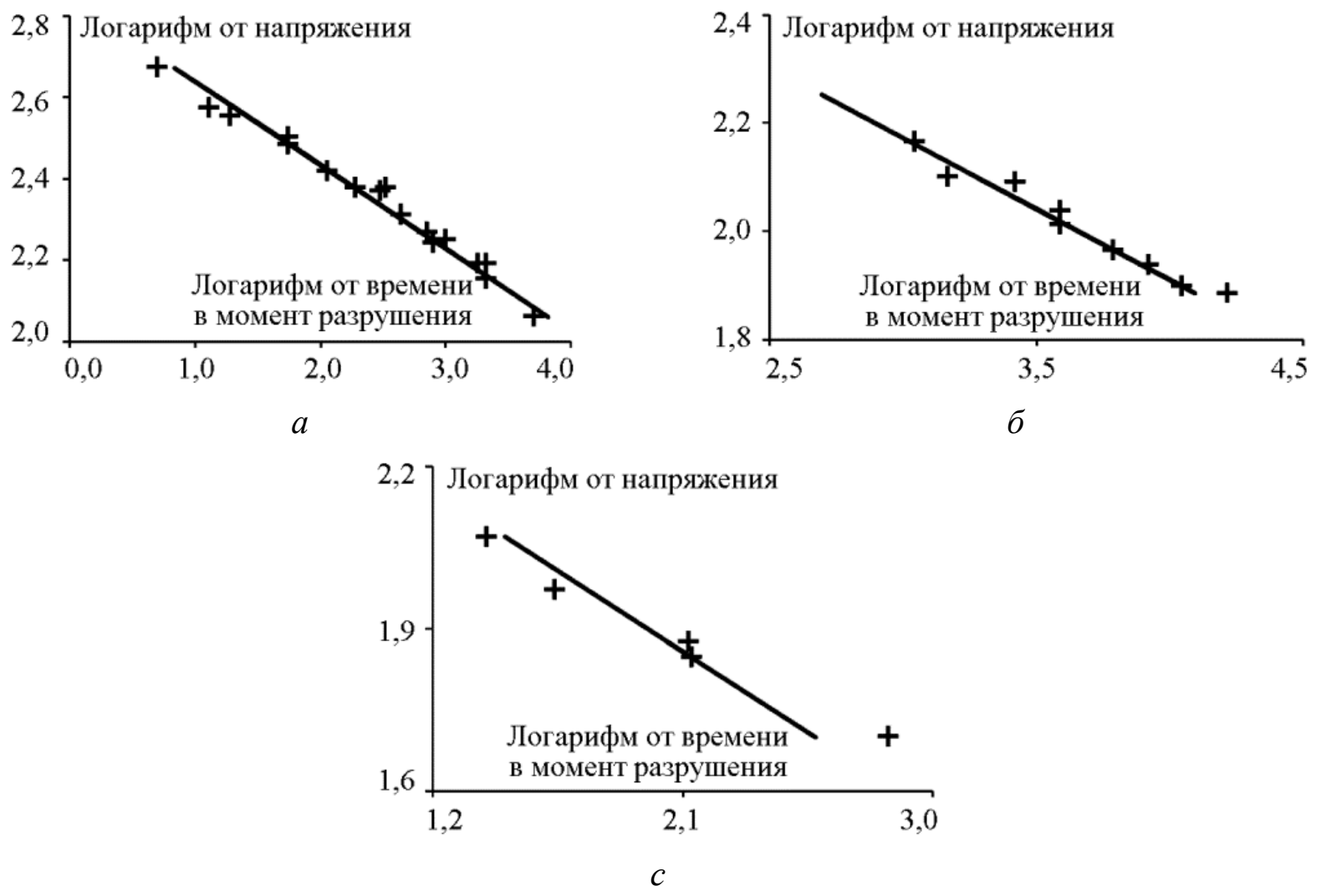

Рис. 1. Зависимости напряжения 1 от времени в момент разрушения: Nimonic 80a при $750{ }^{\circ} \mathrm{C}(a) ; 2,25 \mathrm{Cr}-1 \mathrm{Mo} \mathrm{при} 565^{\circ} \mathrm{C}\left(\right.$ б); медь при $264{ }^{\circ} \mathrm{C}(c)$; крестики - опытные данные;

отрезок прямой - аппроксимация $\lg \left(\frac{\sigma_{\text {inv }}^{1}}{\sigma_{\text {dim }}}\right)=\frac{\lg B_{1}-\lg \left(\frac{t_{\text {rupt }}^{\text {approx }}}{t_{\text {dim }}}\right)}{m_{1}}$ 

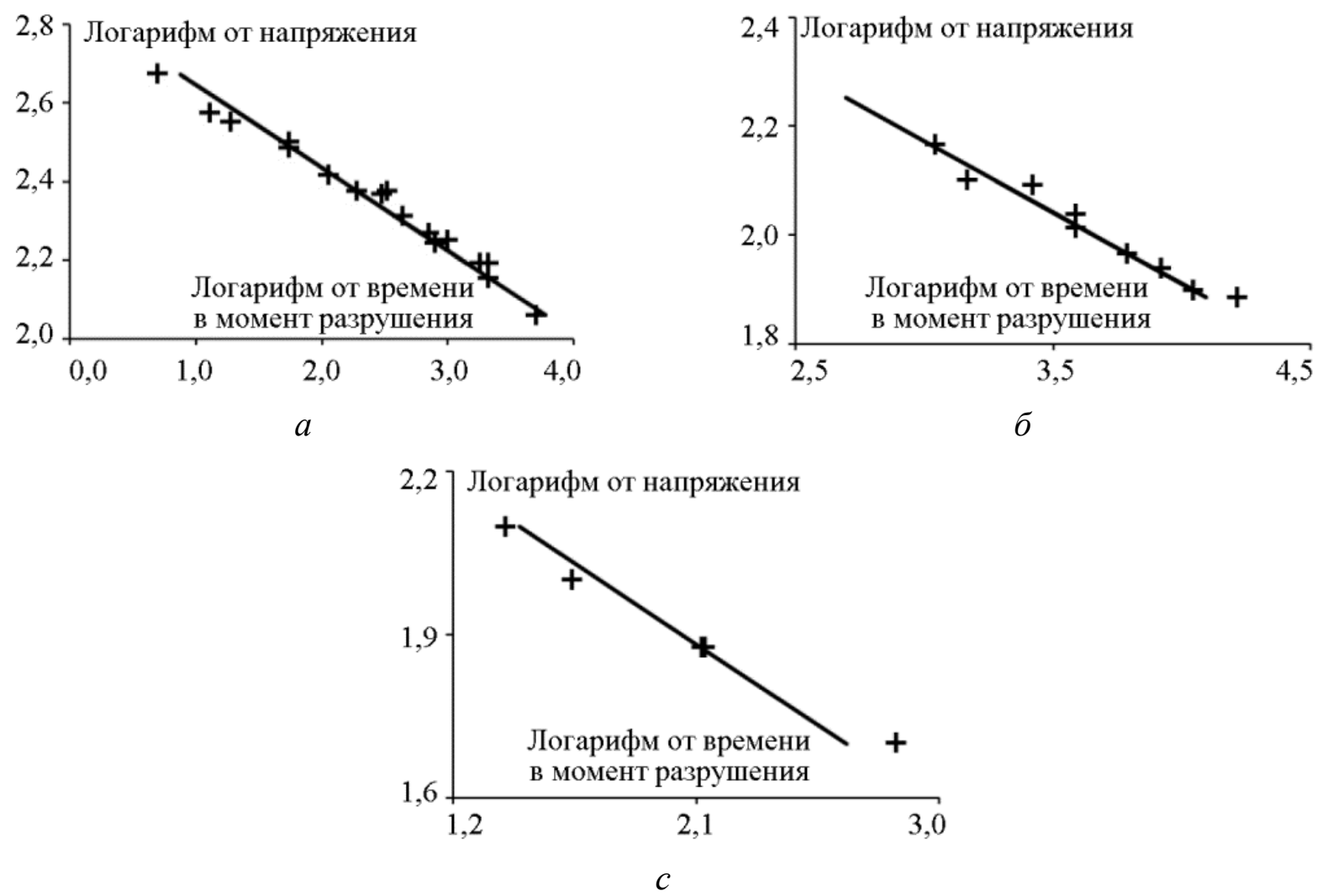

Рис. 2. Зависимости напряжения 2 от времени в момент разрушения: Nimonic 80a при $750{ }^{\circ} \mathrm{C}(a) ; 2,25 \mathrm{Cr}-1 \mathrm{Mo} \mathrm{при} 565{ }^{\circ} \mathrm{C}(б)$; медь при $264{ }^{\circ} \mathrm{C}(c)$; крестики - опытные данные;

$$
\text { отрезок прямой - аппроксимация } \lg \left(\frac{\sigma_{\text {inv }}^{2}}{\sigma_{\text {dim }}}\right)=\frac{\lg B_{2}-\lg \left(\frac{t_{\text {rupt }}^{\text {approx }}}{t_{\text {dim }}}\right)}{m_{2}}
$$

\section{5. Обсуждение}

Из анализа результатов вычислений (табл. 3,4$)$ следует, что отношение $2 \tau_{\max } / \sigma_{\max }$ не определяет влияние максимальных напряжений на время в момент разрушения. Можно предположить, что это влияние может зависеть от механизма процесса ползучести. Механизм межзернового проскальзывания осуществляется под действием $\sigma_{\max }$ при $0 \leq \beta_{2}<0,5$. Механизм перемещения дислокаций внутри зерен реализуется под действием $2 \tau_{\max }$ при $0,5 \leq \beta_{2}<1$. Из анализа погрешностей (табл. 5) следует, что аппроксимация времени в момент разрушения лучше достигается линейными комбинациями 1 и 2 , чем базовыми инвариантами тензора напряжений. Между тем существенного преимущества одной из двух этих моделей не обнаружено.

\section{Литература}

1. Локощенко А. М., Назаров В. В. Кинетический подход исследования длительной прочности металлов при двухосном растяжении // Авиационно-космическая техника и технология. - 2005. - № 10 (26). - С. 73-79.

2. Локощенко А. М., Назаров В. В. Выбор критериев длительной прочности металлов при сложном напряженном состоянии // Авиационно-космическая техника и технология. 2004. - № 7 (15). - C. 124-128. 
3. Lokoshchenko A. M. Estimation of Equivalent Stresses in the Analysis of Long-Term Strength of Metals under Combined Stress State // Mechanics of Solids. - 2010. - Vol. 45, no. 4. P. 633-647. - DOI: 10.3103/S0025654410040126.

4. Lebedev A. A. The theory of equivalent stresses as a problem of mechanics of materials // Strength of Materials. - 1996. - Vol. 28, no. 2. - P. 94-108. - DOI: 10.1007/BF02215833.

5. Dyson B. F., Mclean D. Creep of Nimonic 80A in torsion and tension. Metal Science. 1977. - Vol. 11, iss. 2. - P. 37-45. - DOI: 10.1179/msc.1977.11.2.37.

6. Cane B.J. Creep damage accumulation and fracture under multiaxial stresses // Proc. 5th Int. Conf. Fract., Cannes, 1981. - 1981. - Vol. 3. - P. 1285-93.

7. Nazarov V.V. Determination of creep properties under tension and torsion of copper tubular specimens // Inorganic Materials. - 2014. - Vol. 50, no. 15. - P. 1514-1515. DOI: $10.1134 / \mathrm{S} 0020168514150138$.

8. Назаров В. В. Описания установившейся ползучести при растяжении и кручении трубчатых образцов // Заводская лаборатория. Диагностика материалов. - 2015. - Т. 81, № 7. - С. 60-61. 